\title{
Long-range surface plasmon based tapered fiber optic sensor with enhanced sensitivity using silver nano-layer through radially polarized light
}

\author{
Nabamita Goswami, Kamlesh Kumar Chauhan and Ardhendu Saha* \\ Department of Electrical Engineering National Institute of Technology Agartala \\ Barjala, Jirania, Tripura (West) - 799046, India \\ *Email: nabamita08@rediffmail.com
}

The presented proposal of long rang surface plasmon (LRSP) configuration with tapered fiber structure and radially polarized light beam is a new and different approach towards the sensitivity enhancement of surface plasmon resonance (SPR) based fiber optic sensors. Here the taper waist region of optical fiber having diameter around $400 \mu \mathrm{m}$ is deposited with 690 $\mathrm{nm}$ thick Teflon, $70 \mathrm{~nm}$ thin Ag layer and the sensing layer with refractive index (1.333-1.353) respectively for achieving the dielectric-metaldielectric interface of LRSP configuration which is shown in Figure 1. The cylindrical symmetry and special radial field distribution of radially polarized light make its more interesting SPR study and leads to the enhanced excitation of surface plasmon wave resulting in 4.01 times better sensitivity of fiber optic sensor output response as compared to p-polarized light beam. This sensitivity analysis provides an evidence for theoretical explanation towards the enhanced excitation of LRSP and expedites the new avenues in the field of sensor applications with radially polarized light [1].

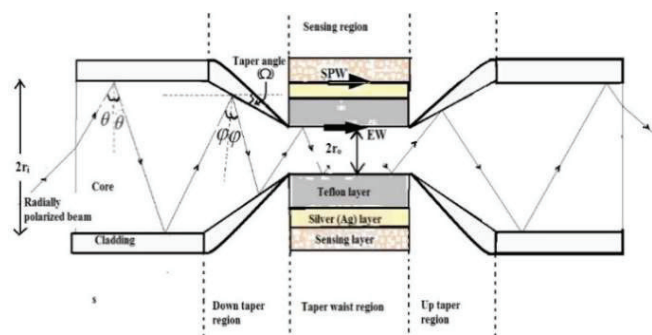

Figure 3: Schematic arrangement of ray tracing in case of tapered multi-mode fiber with LRSP and radially polarized beam

The normalized transmitted power characteristics for the proposed tapered fiber LRSP configuration for varying wavelength of radially polarized beam as required for the sensitivity calculation is shown in Figure 2 exploring in red-shift of the SPR wavelength as increase in refractive index of sensing layer [2].

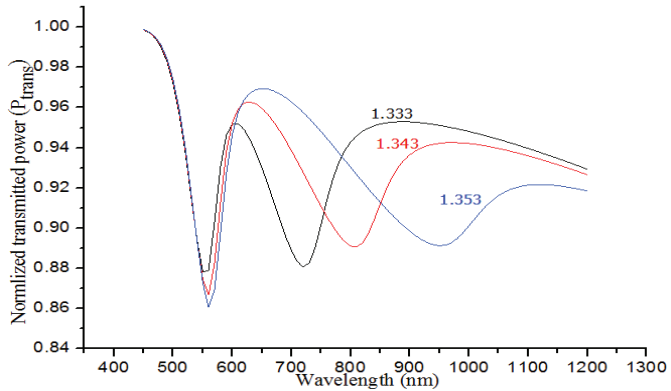

Figure 2: Normalized transmitted power $\left(\mathrm{P}_{\text {trans }}\right)$ spectra for the variation of incident wavelength at different refractive index values $1.333,1.343$ and 1.353

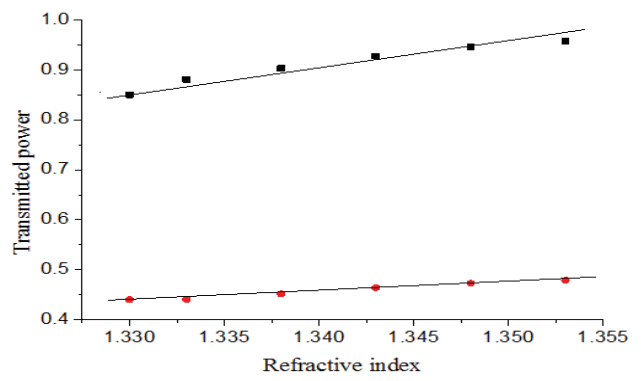

Figure 3: Change in the transmitted power at $720 \mathrm{~nm}$ wavelength for refractive index change, in case of radially and p-polarized light

It is clearly depicted from Figure 3 that, there will be 4.01 times increment in sensitivity of sensor output response in case of excitation of LRSP with radially polarized light as compared to ppolarized light.

\section{References}

1. Y. Maya, N. Cardona, P. Trujillo, Rev. Fac. Ing. Univ. Antioquia N. ${ }^{\circ} 70$ (2014) 167-172.

2. J. Yan, Y. Lu, P. Wang, C. Gu, R Zheng, Y. Chen, H. Ming, Q Zhan, Chinese Optics Letter 7 (2009) 909911. 